\title{
EDITORIAL
}

\section{Military Cardiac Rehabilitation}

Cardiac Rehabilitation may be considered as the return of patients with cardiac disease to an optimal physical, medical, psychological, social, emotional, vocational and economic status $(1,2)$. There have been considerable developments in this process over the last 30 years. Initially it took the form of gradual mobilisation with a return to light physical activity. Now it often involves more demanding physical activity with attempts at psychological repair and secondary prevention.

Formal cardiac rehabilitation programmes usually begin several weeks or months after the myocardial event. The very process however starts much earlier within hours of the cardiac event in the coronary care unit or post-operative ward - with education, mobilisation and reassurance. As recovery progresses the subject will improve, both physically and psychologically, and a formal cardiac rehabilitation programme can then be introduced.

The final aim must be to return subjects to work in a physically and psychologically fit state with a resultant improvement both in quality and quantity of life.

The army has been running such cardiac rehabilitation programmes for 13 years with occasional breaks for operational and staffing reasons. These courses were conceived by the then Colonel I C Crawford L/RAMC, Consultant Cardiologist and Captain D Duncan APTC. These courses are rather unique in the United Kingdom in the form they take. They are three week intensive inpatient courses aimed at relatively young males.

They are inpatient courses because subjects have often to travel considerable distances to take part in courses. The majority of patients are in their 30's or early $40^{\prime}$ 's reflecting the age groups involved in the British Army. They may have had an infarct, developed stable angina which is being treated medically, had an angioplasty or had coronary artery by-pass graft surgery but they share the same basic problem; they have had a psychologically devastating illness for which society wishes to 'wrap them in cotton wool' and protect them for the rest of their life. For these individuals cardiac rehabilitation is the key to a return to normal life.

All patients on these courses are thoroughly assessed. Exercise is an important part of rehabilitation and an exercise test to determine the subject's response is an essential first step. From this initial symptom limited maximal exercise test heart rates for steady state exercise regimen for the three week course are advised $-70 \%$ of maximal heart rate for week one, $75 \%$ for week two and $80 \%$ for week three. All exercises are under the supervision of a qualified physiotherapist and nurse who are the principal members of the rehabilitation team. A variety of exercises are then carried out aiming to maintain the steady state for a period of around 20 minutes after warm up period.

Exercise is not everything in cardiac rehabilitation? Education about heart disease and its risk factors important. Simple lectures on heart disease, diet, smolo․ ing and stress avoidance are among the subjects covereg in discussion sessions. Individuals risk factors are assessed and appropriate advice and intervention of fered. Other members of the rehabilitation team are important at such times and they may include dieticians and psychologists. At the end of the course the individitu al (with their spouse invited) has a formal interview wit? the rehabilitation team, including the physician. Th interview aims to give an assessment of the individual's problems and future treatment. Specific advice on rist factors is offered and any questions the individual hap are answered.

Cardiac rehabilitation does not stop with the form course. The patient must be given encouragement to re turn to a normal life and be supported in the yearsit come with continuing advice and monitoring. Spoise peers, hospital and general practitioner support arefat essential.

Such a course does not come cheap. Three week $\overrightarrow{0} \vec{f}$ inpatient time with a dedicated nurse and physiotherap ist is a precious commodity. It is only correct there that we assess the results of rehabilitation in general.

The success of a formal cardiac rehabilitation gramme must be measured by several factors which should principally include:

1. The ability of the subject to return to work.

2. Improvement in the physical well-being of the if dividual.

3. Improvement in the psychological well-being the individual.

4. An overall reduction in morbidity and mortali of the rehabilitated population.

Each of these factors will be considered in turn.

\section{Return to Work}

Almost without exception our military patients returis to work following rehabilitation. Medical discharge wi a diagnosis of ischaemic heart disease is a rare event. If contrast Shanfield in a review article demonstrated that to $38 \%$ of the general population do not return to wor (3). The age group of patients in the military who attend our rehabilitation programmes is such that they include? high proportion of senior non-commissioned officeng and officers who represent a considerable investment in training and expertise which the Services need to retain Undoubtedly many would return to work without ca formal rehabilitation programme, as some Servicem 
do, but probably not in the same physical or mental state.

This point is illustrated by attitudes in subjects who are undertaking the first rehabilitation courses after a one year pause brought about by operational and staffing problems. Amongst these subjects are those who were unable to attend a course due to its suspension, many of these had not returned to full time duties due to general concern by themselves and their colleagues about their ability to return to duty. The reassurance given in an outpatient interview does not approach that gained in an intensive cardiac rehabilitation programme. All subjects are returned to duties appropriate to their medical grade after their course.

\section{Physical Wellbeing}

The crude measure of an individual's physical wellbeing after a cardiac event is their ability to perform physical exercise. One of the major questions is whether physical exercise capacity improves as a result of a cardiac rehabilitation programme or spontaneously as a result of natural recovery. A study by Goble et al (4) reported in the British Heart Journal demonstrated that light group exercise regimes were as beneficial as heavier aerobic exercises using physical working capacity as assessed by treadmill exercise tests. The patients in this study had a mean age in excess of 50 and were therefore older than the patients in our cardiac rehabilitation courses. Our intensive exercise programme is probably more in line with the expectations of our patients in respect of their previous physical condition, ages and employment demands than light exercise would be. Goble's study also found that of the standard exercise training patients only $65 \%$ attended more than $75 \%$ of available classes and in the light exercise group only $41 \%$ attended three quarters of their classes. The completion rate for our three week intensive course approaches $100 \%$.

\section{Psychological Wellbeing}

It is much harder to quantify any improvement in psychological status. It is a clinical impression by many physicians involved in rehabilitation that there is a substantial improvement and this was stated in published guidelines $(5,6)$. Significant improvements in quality of life indicators were noted in our patients undergoing cardiac rehabilitation after coronary artery bypass graft surgery (7). This is not reflected in the few controlled randomised studies $(8,9)$.

Mayou et al (8) reported little evidence that quality of everyday life was improved between 3 and 18 months after a heart attack with a cardiac rehabilitation programme. Like other non-controlled groups we note a very positive attitude in our rehabilitation patients as reflected in their attitude to return to work as previously discussed.

\section{Morbidity and Mortality}

The military cardiac rehabilitation group has no comparable control and with such an unusual population of young ischaemics it is difficult to comment on any improvement in these parameters. It has been sug- $\overrightarrow{\mathbb{D}}$ gested that physical exercise will encourage collateral circulation and reduce angina. This is obviously difficult 0 to prove in an unmatched group as with physical training o exercise tolerance at lower heart rates will improve thereby increasing exercise ability.

A controlled trial in 1979 (10) showed a reduction in mortality with cardiac rehabilitation but this group also $\frac{\bar{\sigma}}{\bar{c}}$ had more intensive risk factor intervention. Although $\frac{\bar{p}}{\vec{D}}$ reduction in smoking in both groups was equal the in- $\mathbb{\perp}$ tervention group achieved lower blood pressures, serum cholesterols, serum triglycerides and body weights. This $\vec{P}$ is the only major randomised trial of exercise in cardiac? rehabilitation to demonstrate a fall in mortality.

Many other trials have been performed and these are the subject of meta analysis. May et al (11) in 1984 reviewed long term trials and reported a significant re- $\bar{\emptyset}$ duction in risk. Oldridge (12) carried out meta analysis $\frac{\dot{ }}{\omega}$ on 10 randomised trials showing a significant $25 \%$ reduction for cardiovascular death in the control group. Reinfarction was not however reduced. Further meta analysis by O'Connor et al (13) reviewed 22 randomised $\vec{\circ}$ trials and demonstrated a significant $20 \%$ reduction 개글 total and cardiovascular mortality. Again there was significant difference in non fatal reinfarction betweê. the two groups.

Such pooling of data is difficult as there are d⿺𠃊⿻肀二丿 ferences in patient populations with variability \&n? enrolment time after the event, the specific method intervention and geographical features.

\section{Conclusion}

Cardiac rehabilitation attempts to return individuals with heart disease to useful employment in an optimal $\stackrel{\unrhd}{\complement}$ physical and psychological state whilst improving long $\overrightarrow{\hat{O}}$ term morbidity and mortality. Despite 30 or more years 3 of cardiac rehabilitation programmes many questions remain to be answered. Return to work is almost uni-⿱艹 versal in our group and is probably not significantly affected by rehabilitation. Whether people perform better at work is more difficult to test formally, but re-咲 cent patient attitudes after a suspension in our course $\vec{\sigma}$ would suggest there is improvement with a cardiac re-habilitation programme.

Exercise ability improves after a short period of time and contributes to a feeling of physical and psychological well-being even though randomised trials $\frac{\bigcirc}{3}$ shed doubt on whether heavy or light exercise is important.

The final and perhaps most important consideration is $\frac{7}{0}$ the outcome on morbidity and mortality. Meta analysis shows an improvement in mortality in the region of $20-\tilde{N}$ $25 \%$ but not change in non-fatal reinfarction. It is difficult to explain why there is a reduction in mortality but not non-fatal reinfarction. Possible mechanisms evoked ${ }^{\omega}$ include myocardial revascularisation, protection against 
fatal dysrhythmias, improved cardiovascular risk factor profile, improved cardiovascul fitness and increased surveillance. The fact that there is no change in non-fatal reinfarction may represent better survival of a lower infarction rate.

Kallio (10) demonstrated that organised aftercare after myocardial infarction with special emphasis on optimum medical control and health education combined with an exercise programme reduced mortality. This is the very essence of the army's cardiac rehabilitation programme which is designed to meet the demands of its special population. Exercise is an integral part of a soldier's life and must therefore be so in his rehabilitation.

Cardiac rehabilitation is a team effort. It depends upon prompt referral of appropriate patients, sympathetic and positive treatment from the rehabilitation unit staff and the continuing support by hospital and general practitioners to maintain progress. It does return people to duty with a positive attitude to their future and must therefore be considered an asset in the care of these individuals.

N INESON

\section{REFERENCES}

1. Erb B D, Fletcher G F, Sheffield T L. Standards for exercise treatment programs. American Heart Association Sub-Committee on Rehabilitation Target Activity Group. Circulation 1979; 59: 1084A-90A.

2. LIPKIN D P. Is Cardiac Rehabilitation Necessary? Br Heart J 1991; 65: 237-8.

3. Shanfield S B. Return to work after an acute myocardial infarction: a review. Heart Lung 1990; 19: 109-17.
4. Goble A J, Hare D L, MacDonald P S, Oliver, R G, ReID M A, Worcester M C. Effect of early programmes of high and low intensity exercise on physical performance after transmural acute myocardial infarction. Br Heart J 1991; 65: 126-31. 응

5. International Society and Federation of Cardiologye Scientific Councils. Secondary prevention in sur: vivors of myocardial infarction. $\mathrm{Br} \mathrm{Med} \mathrm{J} 1981$ 282: 894-96.

6. AHA Council on Scientific Affairs - Physician supervised exercise programs on rehabilitation of patients with coronary heart disease. JAMA 1981 的 245: $1463-66$.

7. HoAd N A, Crawford I C. Rehabilitation afteen coronary artery by-pass grafting and improved quality of life. BrJSp Med 24: 120-122.

8. Mayou R, MacMahon D, Sleight P, Florencio ML J. Early rehabilitation after myocardial infarction ${ }^{\omega}$ Lancet 1981; 26: 1399-401.

9. STERN M J, Cleary P. The National Exercise and Heart Disease Project: long-term psychologicaధ outcome. Arch Intern Med 1982; 142: 1093-7.

10. Kallio V, Hamalainen M, Hakkila J, LuRILa O Jœ Reduction of sudden deaths by a multifactorial in tervention program after acute myocardial infarc-b tion. Lancet 1979; ii: 1091-4.

11. May G S, Eberlein K A, Friberg C D. Secondagy prevention after myocardial infarction. A review⿳ె口 $\mathrm{f}-$ long-term trials. Prog Cardiovasc Dis 1982; 24: 3 삐드 51.

12. Oldridge N B, Guyatt G M, Fischer ME, Rimpa A. Cardiac Rehabilitation after myocardial farction: combined experience of randomizedv clinical trials. $J A M A$ 1988; 260: 945-50.

13. O'ConNor G T, Buring J E, YusuF $S$ et al. An overview of randomized trials of rehabilitation with exercise after myocardial infarction. Circulation 1989; 80: 234-44.

\section{CORRECTION}

1. Norman Hepple's portrait of Queen Elizabeth the Queen Mother, reproduced in this Journal on her Golden Anniversary as Colonel in Chief, was commissioned by the Herbert Officers' Mess, not the Royal Army Medical Corps.

2. The Queen Elizabeth Military Hospital is named after our Colonel in Chief, not Queen Elizabeth the Second as stated in the same Journal. 